\title{
Directions vs. averages: an in-vivo comparison for cardiac DTI
}

\author{
Andrew D Scott ${ }^{1,2^{*}}$, Pedro Ferreira ${ }^{1,2}$, Sonia Nielles-Vallespin ${ }^{3}$, Laura-Ann McGill ${ }^{2,1}$, Dudley J Pennell ${ }^{1,2}$, \\ David Firmin ${ }^{1,2}$ \\ From 18th Annual SCMR Scientific Sessions \\ Nice, France. 4-7 February 2015
}

\section{Background}

The ability to interrogate cardiac microstructure has led to much recent interest in in-vivo cardiac diffusion tensor imaging (cDTI). However, when compared to studies performed in neuro-imaging, very little work has been done to determine the optimal diffusion encoding schemes. Previous work has suggested that accuracy is improved by increasing the number of diffusion encoding directions $\left(\mathrm{N}_{\mathrm{dirs}}\right)^{1,2}$, but comparisons in the heart have been limited to fixed animal specimens ${ }^{3}$. Here we compare parameters derived from cDTI using data acquired in vivo with an increasing $\mathrm{N}_{\text {dirs. }}$.

\section{Methods}

10 healthy subjects were imaged on a Siemens Skyra using the STEAM-EPI cDTI sequence ${ }^{4}$ in a short-axis slice of the mid left-ventricle with the optimal protocol recently described $^{5}$ (b-values: 150 and $750 \mathrm{smm}^{-2}, 2.8 \times 2.8 \times 8 \mathrm{~mm}^{3}$ resolution). This was repeated with $\mathrm{N}_{\text {dirs }}=6,10,12$ and 20 (standard Siemens product directions) and 12 averages $\left(\mathrm{N}_{\mathrm{avs}}\right)$ were acquired in each direction. The diffusion tensor and parameter maps including mean diffusivity (MD), helical angle (HA) and fractional anisotropy (FA) were calculated as previously described ${ }^{5}$, using all averages and all directions together to provide a reference data set. The processing was then repeated for each set of diffusion encoding directions with varying numbers of averages chosen to match the total images used $\mathrm{N}_{\text {tot }}=\mathrm{N}_{\text {avs }} \times \mathrm{N}_{\text {dirs }}$, as closely as possible to 24,36 and 60 and also using $\mathrm{N}_{\mathrm{avs}}=12$.

\section{Results}

Figure 1 shows example parameter maps (HA, MD and FA) calculated using all directions $\left(\mathrm{N}_{\mathrm{dir}}=48\right)$ and all averages $\left(\mathrm{N}_{\mathrm{av}}=12\right)$ together compared to each diffusion encoding scheme processed with $\mathrm{N}_{\text {tot }}=60$. There was no consistently visible difference between the encoding schemes. Figure 2 shows mean MD and FA values for each $\mathrm{N}_{\text {dirs }}$ plotted with the $\mathrm{N}_{\text {tot }}$ and the average variation (standard deviation) in these parameter maps over the left ventricle. For a given $\mathrm{N}_{\text {tot }}, \mathrm{N}_{\text {dirs }}=10$ appears to have the minimum variation of FA across the left ventricle and most commonly has FA closest the reference value and $\mathrm{N}_{\text {dirs }}=12$ appears to be superior when considering MD. However, a comparison of MD and FA values when $\mathrm{N}_{\text {tot }}=60$, showed no statistically significant difference between $\mathrm{N}_{\text {dirs }}$ (1-way repeated measures ANOVA; MD: $\mathrm{p}=0.59$; FA: $\mathrm{p}=0.82)$.

\section{Conclusions}

While simulations in previous work have found increasing $\mathrm{N}_{\text {dirs }}$ to result in more accurate results, our results suggest that any resultant changes in MD or FA measured in in-vivo myocardium are small.

\section{Funding}

This work was performed at The National Institute for Health Research Funded Cardiovascular Biomedical Research Unit at The Royal Brompton Hospital and Imperial College London. 

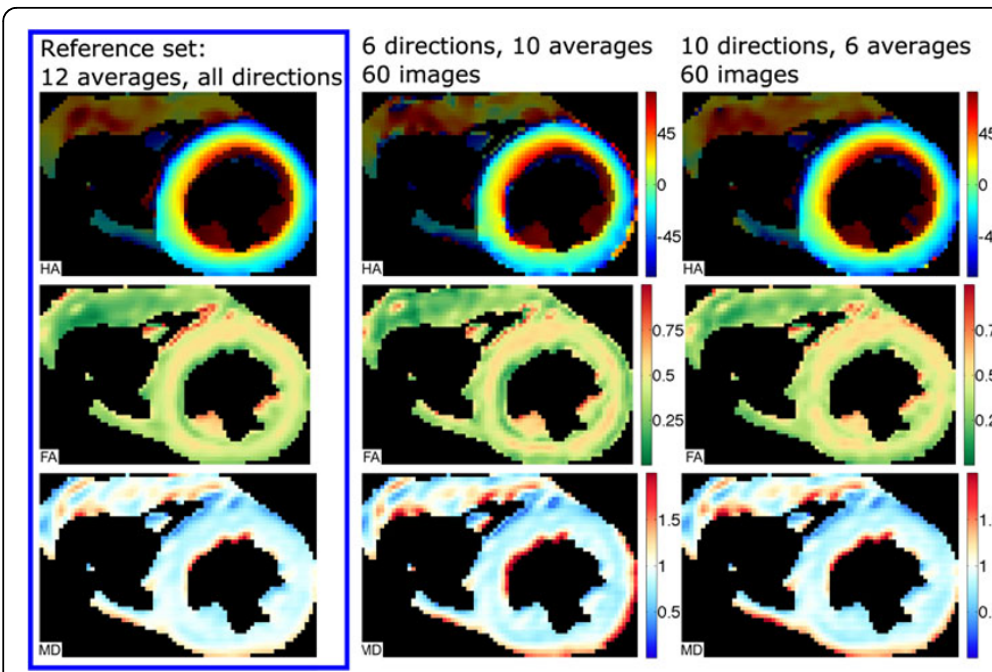

12 directions, 5 averages 60 images

20 directions, 2 averages 60 images

60 images

Figure 1 Maps of helical angle (top row), fractional anisotropy (middle) and mean diffusivity (bottom row) for one example healthy subject. Maps were calculated for one reference data set (left hand column) with all available data and then with combinations of $N_{\text {dirs }}$ and $N_{\text {av }}$ to total $N_{\text {tot }}=60$ in each case. No consistent changes in parameter map were observed when altering $N_{\text {dirs. }}$.
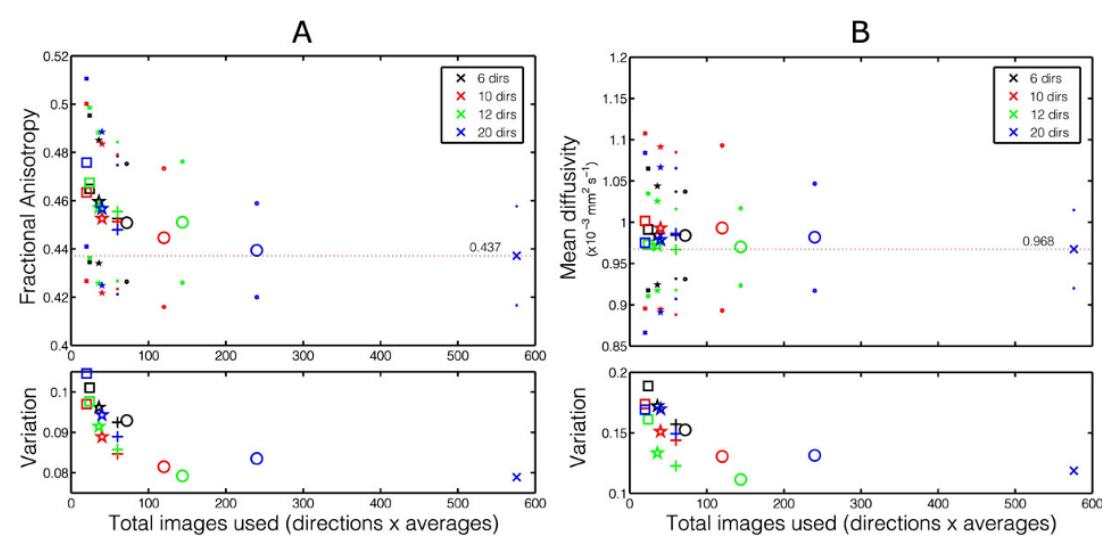

Figure 2 Mean values of fractional anisotropy (A) and mean diffusivity (B) for all 10 subjects (smaller points indicate standard deviations) in the left ventricle, colour coded by the $N_{\text {dirs }}$ and plotted with the $N_{\text {tot }}$ used in the tensor calculation. The horizontal lines indicate the mean values obtained from the reference data set which used every encoding direction and $\mathrm{N}_{\mathrm{av}}=12$. The lower subplots show the standard deviation in the left ventricle averaged over all 10 subjects to provide some indication of the variability of the parameters within a subject. For a given $N_{\text {tot, }}$ the data acquired with $\mathrm{N}_{\text {dirs }}=10$ directions appears to most commonly have the least variation and the FA closest to the reference value. For MD, $N_{\text {dirs }}=12$ appears to be optimal.

\section{References}

1. Jones: MRM 2004, DOl:10.1002/mrm.20033.

2. Ennis: MIA 2014, DOI: 10.1016/j.media.2013.10.009.

3. Mazumder: JCMR 2014, doi:10.1186/1532-429X-16-S1-P359.

4. Nielles-Vallespin: MRM 2012, DOl:10.1002/mrm.24488.

5. Scott: MRM 2014, DOl:10.1002/mrm.25418.

doi:10.1186/1532-429X-17-S1-P25

Cite this article as: Scott et al.: Directions vs. averages: an in-vivo

comparison for cardiac DTI. Journal of Cardiovascular Magnetic Resonance 2015 17(Suppl 1):P25

\section{Submit your next manuscript to BioMed Central} and take full advantage of:

- Convenient online submission

- Thorough peer review

- No space constraints or color figure charges

- Immediate publication on acceptance

- Inclusion in PubMed, CAS, Scopus and Google Scholar

- Research which is freely available for redistribution 